\title{
I'hypertension artérielle du sujet âgé attitude en odontologie
}

RÉSUMÉ La prévalence de l'hypertension artérielle du sujet âgé est élevée (40 à $52 \%$ selon les auteurs). Systolo-diastolique ou plus fréquemment systolique pure, elle est un facteur de risque cardio-vasculaire majeur.

Par le traitement antihypertenseur mis en place, le taux de morbidité et la mortalité cardio-vasculaire sont en baisse, il est donc nécessaire de traiter l'hypertension artérielle du

Hélène ARNAL

Assistante Hospitalo-Universitaire.

Claude Bernard WIERZBA

M.C.U.-P.H.

Département de pathologie,

chirurgie buccale,

anesthésie, réanimation,

Faculté de Chirurgie Dentaire

Paris-Descartes.

sujet âgé.

L'odontologiste devra bien connaître son patient hypertendu, les traitements antihypertenseurs institués et tous les effets secondaires relatifs aux traitements avant de pratiquer un acte de chirurgie buccale.

\section{MOTS CLÉS}

H.T.A.

pression artérielle

diurétiques

inhibiteurs de l'enzyme de conversion

inhibiteurs calciques

bétabloquants

antihypertenseurs centraux

effets secondaires 


\section{introduction}

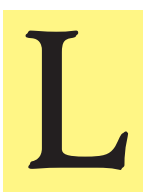

es données épidémiologiques relient de façon incontestable l'incidence de l'hypertension artérielle (HTA) avec l'âge. Dans les pays industrialisés, $50 \%$ des personnes âgées de plus de
65 ans souffrent d'HTA, et ceci quelle que soit l'appartenance ethnique ou le sexe. Il s'agit donc d'une manifestation physiologique de l'avancée en âge, résultant de la combinaison de facteurs environnementaux et génétiques.

\section{physiopathologie de I'HTA du sujet âgé}

La pression artérielle moyenne dépend du débit cardiaque et de la résistance à la déformation des parois artérielles.

D'une façon générale, un sujet est considéré comme hypertendu lorsque sa pression artérielle systolique est supérieure à $140 \mathrm{mmHg}$ et sa pression artérielle diastolique est supérieure à $90 \mathrm{mmHg}$.

Alors que chez le sujet jeune l'augmentation de la tension artérielle s'explique davantage par celle du débit cardiaque, chez le sujet âgé l'origine en est très souvent l'accroissement de la résistance artérielle.

\section{- HTA primitive}

Elle représente $95 \%$ des HTA et répond à certains facteurs de risque : âge, surcharge pondérale, excès de consommation de sel, antécédents familiaux. Elle est encore assez mal expliquée ; il existe plusieurs hypothèses :

\section{LE SYSTÈME NERVEUX SYMPATHIQUE}

Certains sujets développeraient une hyper-réactivité du système nerveux sympathique. Il aurait une action directe en augmentant la fréquence cardiaque, donc son débit, et en augmentant la vasoconstriction, donc la résistance artérielle. Il aurait une action indirecte en modifiant la réponse $d u$ rein aux variations de la volémie.

\section{LE SYSTÈME \\ RÉNINE-ANGIOTENSINE- ALDOSTÉRONE}

L'aldostérone est une hormone hypertensive : par une action sur la pompe $\mathrm{Na}+/ \mathrm{K}+$ l'aldostérone augmente la réabsorption de sodium, donc la réabsorption d'eau, ce qui augmente la volémie. On observe que les hypertendus ont très fréquemment une dérégulation de ce système.

\section{VIEILLISSEMENT DE LA STRUCTURE DE LA PAROI ARTÉRIELLE}

Il se traduit par une moindre adaptabilité de ces vaisseaux à amortir la pression sanguine, une moindre compliance, une augmentation des résistances artérielles périphériques, et une hyperpulsatilité artérielle. 
Ce facteur est en grande partie à l'origine de l'augmentation de la pression systolique du sujet âgé.

\section{- HTA secondaire}

Elles sont beaucoup moins fréquentes que les HTA primitives, mais correspondent souvent à des formes graves. Le traitement étiologique, lorsqu'il est possible, en permet la guérison.

- Origine médicamenteuse ou toxique

Traitements médicamenteux par hormones oestroprogestatives de synthèse, glucocorticoïdes, AINS, ciclosporine, antidépresseurs IMAO, sympathico-mimétiques, etc.

Intoxication alcoolique à la cocaïne. Consommation régulière de réglisse.

- Origine rénale

- Étiologie hormonale

Dysfonctionnement de la glande surrénale :
- syndrome de Conn : hypersécrétion d'aldostérone par la glande surrénale dans son ensemble ;

- syndrome de Cushing : hypersécrétion de cortisol par la corticosurrénale ;

- phéochromocytome : hypersécrétion d'adrénaline par la médullosurrénale.

Dysthyroïdie

Acromégalie

\section{- Atteintes artérielles}

Coarctation aortique : rétrécissement $\mathrm{du}$ diamètre de l'aorte, du fait de la présence d'un obstacle dans sa lumière, qui provoque une hyperpression (HTA) dans l'aorte et au niveau des artères des membres supérieurs.

\section{- Liée à un désordre neurologique}

Hypertension intracrânienne, syndrome d'apnée du sommeil, dysautonomie familiale.

\section{complications de I'HTA}

- à court terme : la crise aiguë hypertensive

Elle correspond à une élévation rapide et marquée de la pression artérielle qui pourra atteindre et dépasser des valeurs telles que $25 / 13 \mathrm{cmHg}$.

Elle peut survenir chez un patient dont l'hypertension est traitée, comme chez un patient non diagnostiqué.

Elle peut être causée par :

- une situation très anxiogène ;

- un syndrome douloureux aigu ou chronique ;
- un syndrome fébrile ;

- un sevrage médicamenteux brutal chez un hypertendu chronique ;

- une intoxication par un agent exogène (ciclosporine, sympathicomimétique, antidépresseur tricyclique, AINS, érythropoïétine, cocaïne) ;

- une interaction médicamenteuse diminuant l'effet hypotenseur du traitement ;

- des atteintes artérielles, notamment au niveau aortique ;

- une glomérulonéphrite aiguë ;

- un phéochromocytome. 
Cas particulier de la femme enceinte : une éclampsie qui est une crise hypertensive qui complique la toxémie gravidique au $3^{\text {e }}$ mois de grossesse.

Quel que soit le mécanisme initial, l'élévation de la tension sera d'autant plus marquée qu'elle sera moins amortie par des artères moins compliantes. Les sujets âgés seront donc plus exposés à ce type de crise et leur gravité sera plus importante.

\section{LA POUSSÉE HYPERTENSIVE}

La crise hypertensive peut ne pas entraîner de signes de souffrance viscérale et est alors qualifiée de poussée hypertensive.

La poussée hypertensive peut être asymptomatique ou bien associée à des épistaxis, des vertiges légers, des acouphènes, des céphalées tolérables.

Parfois de multiples poussées sont responsables de petits AVC successifs qui auront au final une répercussion non négligeable sur les fonctions cérébrales.

\section{LES URGENCES HYPERTENSIVES}

La crise hypertensive peut aussi être à l'origine de différents types d'urgences hypertensives :

- une encéphalopathie hypertensive : elle se traduit par l'installation d'un œdème cérébral responsable d'une hypertension intra-crânienne. Les symptômes sont des céphalées, nausées, vomissements, puis agitations, confusion, troubles visuels et enfin convulsions, perte de connaissance et coma ;

- un accident vasculaire cérébral hémorragique ou ischémique, qui se manifeste par des céphalées et des troubles de la conscience ;

- un œdème aigu du poumon, urgence vitale fréquente chez le sujet âgé, qui donne une sensation d'étouffement ;

- une crise coronarienne ;

- une dissection aortique : rupture longitudinale de la média de l'aorte (voir schéma), qui se divise en deux canaux ; le risque est sa rupture avec hémorragie interne.

\section{- I'hypertension artérielle} sévère

Elle se caractérise par une PAS > $180 \mathrm{mmHg}$ et une PAD > $110 \mathrm{mmHg}$ qui ne font pas suite à une élévation brutale.

Elle survient chez l'hypertendu non ou mal équilibré et/ou lors de périodes particulièrement stressantes chez ces patients.

\section{à long terme : retentissement vasculaire}

L'HTA favorise l'athérosclérose : l'athérome est une lésion intimale non uniforme de la paroi artérielle qui se caractérise par l'accumulation d'acides gras, de cholestérol, de tissu fibreux, de cellules inflammatoires et de calcifications. La formation de la plaque d'athérome est aussi la conséquence de l'accumulation des LDL (Low Density Lipoproteins qui transportent le cholestérol) en excès sur les parois artérielles lorsque la capacité de dégradation des LDL par le foie est débordée. Les macrophages vont alors les phagocyter et, chargés de cholestérol, deviennent des cellules spumeuses. 


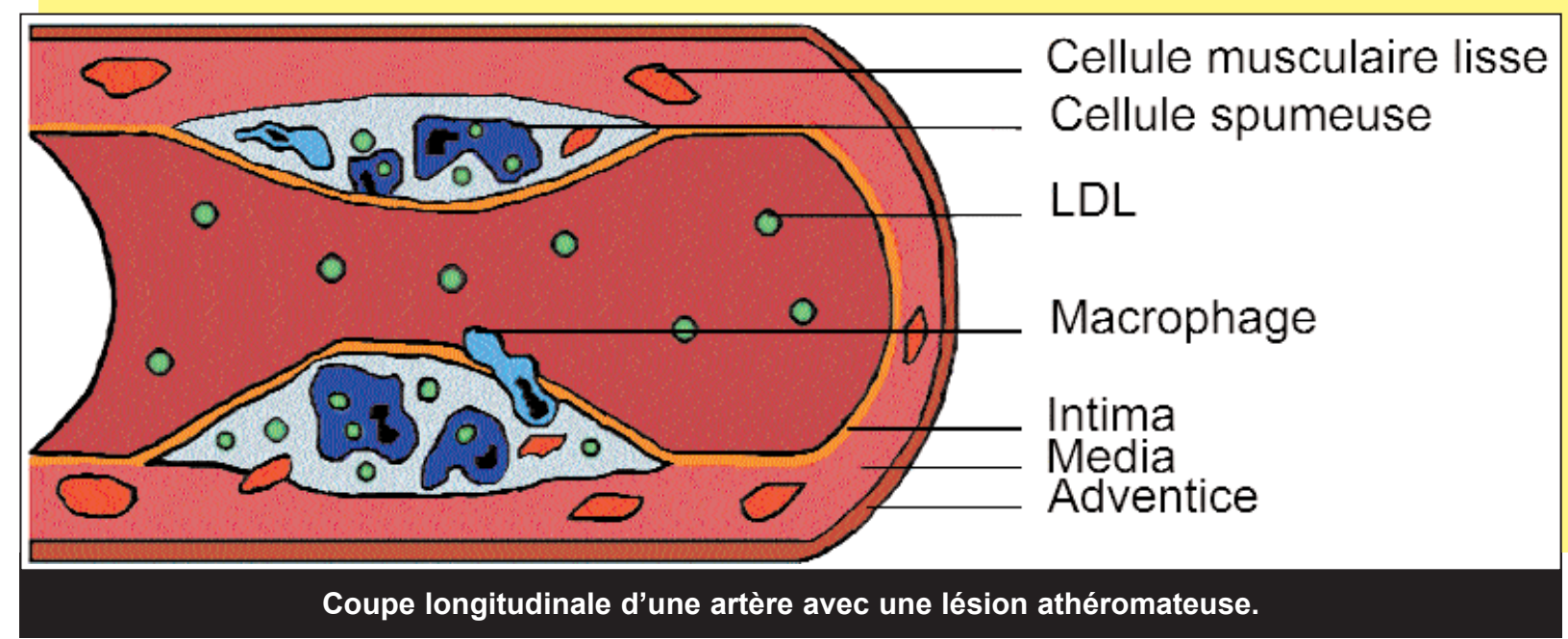

L'athérosclérose peut avoir différentes conséquences :

- La formation et/ou le détachement d'un thrombus par rupture ou érosion de la plaque d'athérome peut entraîner :

- au niveau des artères carotidiennes et cérébrales, un AVC ischémique ;

- au niveau des artères coronaires, une crise d'Angor ou un infarctus ;

- au niveau des artères pulmonaires, une embolie pulmonaire ;

- au niveau des artères des membres inférieurs des crampes à la marche, une artériopathie des membres inférieurs qui peut se compliquer en angiodermite nécrotique (ulcère de la jambe).

- La plaque d'athérome va aussi favoriser la formation d'un anévrisme.

\section{- retentissement cardiovasculaire}

L'HTA va se traduire par une augmentation de la pression dans les artères (postcharge) par rapport à la pression intra-ventriculaire.

\section{CARDIOPATHIE ISCHÉMIQUE}

- L'augmentation de la postcharge crée une résistance à l'éjection du sang par le ventricule gauche (éjection systolique). Le ventricule gauche doit alors fournir un surcroît de travail, donc augmenter sa consommation en oxygène. Ceci potentialise le risque d'insuffisance coronaire.

Ainsi l'HTA multiplie par 3 le risque de cardiopathie ischémique et d'artérite, et multiplie par 5 le risque d'infarctus du myocarde.

\section{HYPERTROPHIE VENTRICULAIRE}

Le surcroît de travail effectué par le ventricule conduit à un épaississement de ses parois : c'est l'hypertrophie ventriculaire.

Les conséquences en sont :

- l'hypertrophie du ventricule gauche diminue son élasticité et sa capacité à se relâcher. Ceci induit une augmentation de la pression capillaire pulmonaire avec dyspnée d'effort : c'est l'insuffisance cardiaque diastolique ;

- elle est à l'origine de troubles du rythme paroxystique avec risque de mort subite. 
INSUFFISANCE CARDIAQUE

Résultant des deux complications précédentes.

- retentissement cérébral

ACCIDENT VASCULAIRE CÉRÉBRAL (AVC)

L'HTA multiplie par 8 le risque d'AVC :

- AVC ischémique en rapport avec les plaques d'athérome carotidiennes ;

- AVC hémorragique par rupture d'anévrisme cérébral.

\section{L'ENCÉPHALOPATHIE HYPERTENSIVE}

C'est une urgence hypertensive (voir cidessus).

\section{retentissement rénal}

L'HTA induit des lésions artériolaires au niveau du rein (néphroangiosclérose) qui peuvent évoluer vers l'insuffisance rénale (diagnostic par le dosage de la créatinine plasmatique). L'atteinte du rein aggrave à son tour l'HTA.

- retentissement au niveau de la rétine

Une thrombose de l'artère ou de la veine centrale de la rétine peut engendrer un accident vasculaire oculaire.

Une rétinopathie hypertensive se diagnostique avec un fond d'œil.

\section{mesure de la pression artérielle}

- la mesure au repos chez le médecin traitant

La mesure doit être prise après 5-10 minutes de repos assis ou allongé. Le brassard est placé au-dessus du pli du coude, le stéthoscope posé sur l'artère humérale. La mesure est réalisée sur les deux bras, en répétant à une minute d'intervalle deux fois la mesure du côté où la PA est la plus élevée. Deux consultations espacées de plusieurs semaines sont nécessaires pour apporter un diagnostic.

- la mesure ambulatoire de la pression artérielle (MAPA)

Le patient est équipé d'un appareil automatique qui va se déclencher à intervalles fixes pendant $24 \mathrm{~h}$; il garde le brassard en place pendant tout ce temps.

Elle est indiquée dans le cadre du suivi d'une HTA lorsqu'on suspecte des épisodes d'hypotension chez un hypertendu traité, pour vérifier l'efficacité d'un traitement sur $24 \mathrm{~h}$, ou lorsqu'une HTA semble résister au traitement pour débusquer un éventuel effet «blouse blanche», notamment chez le sujet âgé.

- I'automesure simple de la pression artérielle

Le patient, préalablement éduqué, doit mesurer sa pression artérielle à intervalles réguliers.

Il utilise un tensiomètre au poignet automatique, facile à manipuler. 
Cette méthode est particulièrement bénéfique pour le sujet âgé dont la pression artérielle est relativement variable. Dans le cadre du suivi de l'HTA, le patient pourra contrôler lui-même plusieurs fois par semaine sa tension. En impliquant davantage le patient, l'automesure aide à l'observance du traitement. En outre, le patient peut alerter son médecin en cas de poussée hypertensive, ce qui limite considérablement la survenue de complications.

\section{traitement}

On se heurte à des difficultés d'observance du fait de l'absence de symptomatologie.

\section{- indications}

De nombreux essais thérapeutiques ont prouvé le bénéfice apporté par les traitements antihypertenseurs dans la prévention cardio-vasculaire; en particulier chez le sujet âgé, il est observé une diminution des complications vasculaires cérébrales de $36 \%$, des coronaropathies de $25 \%$, et de la mortalité totale de $12 \%$.

La Haute Autorité de Santé (HAS) considère les patients hypertendus de plus de 65 ans comme étant «à risque élevé» de complications cardiovasculaires et cérébrales. Les recommandations de 2000 posent de ce fait l'indication presque systématique du traitement de l'hypertendu de plus de 65 ans, à partir du moment où l'hypertension est diagnostiquée de façon certaine.

\section{- règles hygiéno-diététiques}

- Arrêt du tabac (le tabac entraîne une augmentation de la PA de 5 à 10 mmHg dans les 15 à 30 minutes qui suivent la consommation d'une cigarette).
- Consommation de sel $\leq 6 \mathrm{~g}$ par jour.

- Consommation de vin $\leq 2$ verres de vin par jour.

- Activité physique trois fois par semaine.

- Réduction du surpoids, des apports en lipides et en hydrates de carbone.

- Contrôle médical des autres facteurs de risque cardiovasculaire : diabète, cholestérolémie.

\section{traitement médicamenteux}

En première intention, on privilégiera une monothérapie. Une bithérapie peut aussi être envisagée, autorisant des doses réduites du fait de la synergie d'action de certaines spécialités et permettant aussi de baisser les effets secondaires de chacune.

\section{LES BÊTABLOQUANTS}

Les bêtabloquants entraînent une baisse de la pression artérielle en inhibant les bêta-récepteurs cardiaques (par une baisse de la fréquence cardiaque) et en inhibant les bêta-récepteurs des cellules glomérulaires (par une baisse de la sécrétion d'aldostérone, hormone hypertensive). 
Ils sont indiqués en cas de cardiopathie ischémique.

Leurs contre-indications sont l'insuffisance cardiaque, une bradycardie inférieure à $50 \mathrm{btm} / \mathrm{mn}$, l'insuffisance respiratoire, l'asthme, le syndrome de Raynaud.

Leurs effets indésirables sont des chutes tensionnelles, des hypoglycémies.

\section{LES DIURÉTIQUES}

$\mathrm{Au}$ début de leur administration, ils augmentent la natriurèse (évacuation du sodium dans les urines) et entrầnent une perte hydrique concomitante, donc une baisse de la volémie. Le débit cardiaque est alors réduit. Ce mécanisme subit au long cours une compensation physiologique par adaptation $d u$ rein.

Ils ont aussi une deuxième action : ils diminuent la réactivité vasculaire aux vasoconstricteurs physiologiques, ce qui aboutit à une vasodilatation périphérique, responsable de l'effet hypotenseur au long cours.

Il en existe différentes familles : diurétiques thiazidiques et apparentés, diurétiques de l'anse de Henlé, diurétiques bloqueurs de l'aldostérone.

Leurs effets indésirables sont un risque d'hypokaliémie, d'hyponatrémie, de déshydratation, d'hyperglycémie. Ils requièrent une surveillance biologique attentive.

\section{LES INHIBITEURS CALCIQUES}

Ils ont montré leur efficacité dans le traitement de l'HTA systolique du sujet âgé.

Ils bloquent les canaux calciques lents empêchant l'entrée de calcium dans la cellule musculaire, limitant donc sa contraction. Au niveau des cellules musculaires lisses artérielles, la relaxation musculaire entraîne une vasodilatation. Au niveau des cellules myocardiques, ils inhibent la force de contraction.

Leur effet indésirable est un risque d'œdème des membres inférieurs.

\section{LES INHIBITEURS DE L'ENZYME DE CONVERSION (IEC)}

L'inhibition de l'enzyme de conversion de l'angiotensine baisse la concentration en angiotensine II ; l'angiotensine II stimule d'une part la synthèse d'aldostérone, hormone hypertensive et a, d'autre part, une action artériolaire vasoconstrictrice.

En outre, l'enzyme de conversion inhibe la dégradation de la bradykinine, substance vasodilatatrice et natriurétique.

Ils sont indiqués chez les sujets présentant une cardiopathie ischémique, une insuffisance cardiaque, une pathologie rénale (sans insuffisance), un diabète. Ils sont contre-indiqués en cas d'insuffisance rénale.

\section{LES ANTAGONISTES DES RÉCEPTEURS AR II DE L'ANGIOTENSINE (ARAII)}

En bloquant les récepteurs de l'angiotensine II, ils entraînent l'inhibition de la synthèse d'aldostérone et une vasodilatation.

\section{LES ALPHA-BLOQUANTS SÉROTONINERGIQUES}

Par le blocage des récepteurs alpha 1 adrénergiques vasculaires, ils entraînent une vasodilatation artériolaire. 
- médicaments dont l'action s'effectue au niveau du système nerveux central

\section{LES ANTIHYPERTENSEURS CENTRAUX}

Ils agissent en diminuant le tonus sympathique et en augmentant le tonus vagal par stimulation des récepteurs alpha 2 adrénergiques centraux. Ils entraînent une diminution de la fréquence cardiaque, une vasodilatation et une diminution de l'activité du système rénine-angiotensine.

Leurs effets indésirables sont un risque de rebond tensionnel, de somnolence.

Il est primordial de ne jamais arrêter brutalement un traitement antihypertenseur.

\section{conduite à tenir en odontostomatologie}

- prévention

On peut craindre tout d'abord au fauteuil la survenue d'une crise hypertensive aiguë entraînant une urgence hypertensive.

Une poussée hypertensive entraînant une sensation de malaise (épistaxis, vertiges, acouphènes, céphalées) n'est non plus souhaitable pour personne.

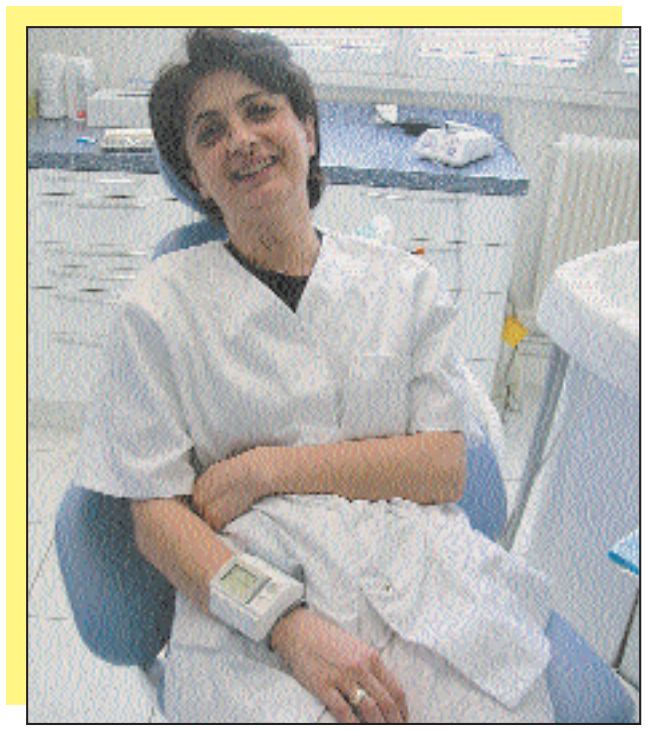

En chirurgie buccale il existe aussi un risque hémorragique, sur une poussée hypertensive simple ou sur une élévation de la tension moins ponctuelle mais bien présente.

En premier lieu le questionnaire médical dépistera les patients hypertendus et leur traitement. Un contact avec le médecin traitant peut s'avérer nécessaire pour s'assurer que l'HTA et les éventuelles autres pathologies associées 
sont bien équilibrées. Aucun acte chirurgical ne devrait a priori être entrepris sur une hypertension non stabilisée.

Puis, pour chaque patient hypertendu, même bien équilibré, on devrait procéder à un contrôle de sa pression artérielle avant tout acte chirurgical: il existe des tensiomètres au poignet très fiables et en vente dans toute pharmacie à coût modique.

Enfin, pour limiter l'anxiété et rendre les séances plus confortables, il est souhaitable, particulièrement chez ces patients, de préférer des séances courtes, programmées le matin, et de prescrire une prémédication sédative.

\section{- respect des interactions médicamenteuses}

Nous ne considèrerons ici que les interactions avec les spécialités prescrites en odontostomatologie.

\section{BÊTABLOQUANTS}

Ils interagissent avec :

- les AIS qui entraînent une rétention hydrosodée au long court et réduisent l'effet hypotenseur ;

- les AINS qui réduisent l'effet hypotenseur en inhibant les prostaglandines vasodilatatrices ;

- la floctafénine (antalgique) : contreindication absolue ;

- les topiques intestinaux qui diminuent l'absorption digestive du bêtabloquant.

\section{DIURÉTIQUES}

Ils interagissent avec :

- les AIS qui entraînent une rétention hydrosodée au long court et réduisent l'effet hypotenseur ;
- les AINS qui réduisent l'effet hypotenseur et peuvent avoir un retentissement sur le rein : en diminuant la filtration glomérulaire par inhibition de la synthèse des $P G$ vasodilatatrices ils peuvent entraîner une insuffisance rénale qui peut être aiguë si le sujet est déshydraté.

\section{LES INHIBITEURS CALCIQUES}

Ils interagissent avec :

- les AIS qui réduisent l'effet hypotenseur ;

- l'itraconazole et le kétoconazole (antifongiques) qui majorent les effets indésirables des inhibiteurs calciques : il y a une compétition au niveau du métabolisme hépatique qui entraîne un risque d'œdème (sauf pour le vérapamil et diltiazem).

\section{LES INHIBITEURS DE L'ENZYME DE CONVERSION (IEC) \\ LES ANTAGONISTES \\ DES RÉCEPTEURS AR II \\ DE L'ANGIOTENSINE (ARAII)}

Ils interagissent avec :

- les AIS qui réduisent l'effet hypotenseur ;

- les AINS qui réduisent l'effet hypotenseur et peuvent entraîner une insuffisance rénale aiguë chez le sujet prédisposé (en diminuant la filtration glomérulaire par inhibition de la synthèse des PG vasodilatatrices).

\section{LES ALPHA-BLOQUANTS \\ SÉROTONINERGIQUES}

Interaction avec les AIS qui réduisent l'effet hypotenseur. 


\section{LES ANTIHYPERTENSEURS CENTRAUX}

Interaction avec les AIS qui réduisent l'effet hypotenseur.

Du fait d'un risque majoré de neurosédation, contre-indication à la prescription concomitante d'autres dépresseurs du SNC (prémédications sédatives: hydroxyzine, benzodiazépines).

- manifestations buccales liées aux effets indésirables des traitements hypotenseurs

\section{HYPOSIALIE}

Elle peut être provoquée par certains diurétiques, antihypertenseurs centraux, alpha-bloquants, inhibiteurs calciques et est réversible à l'arrêt du traitement.

Ces médicaments peuvent être à l'origine d'une sialose médicamenteuse : atteinte non inflammatoire, qui se traduira par une hyposialie et, très souvent, par un gonflement bilatéral indolore des glandes salivaires, notamment des parotides.

Ces médicaments peuvent aussi provoquer une inflammation des glandes salivaires (sialite).

Si le traitement ne peut pas être aménagé, il faudra recommander une hydratation fréquente, une hygiène stricte et d'éviter les aliments secs, l'alcool et le tabac. Des moyens de stimulation pourront être conseillés, comme les gommes à mâcher. Une prescription de pulvérisations endo-buccales avec artisial ${ }^{\circledR}$ permet souvent de stimuler une sécrétion salivaire transitoire.
Les traitements par cholinergiques sialagogues (éséridine, teinture de Jaborandi diluée au $5^{\mathrm{e}}$ ) et les adrénolytiques (dihydroergotamine) peuvent être essayés en cas d'échecs des premiers. Le recours à l'anétholtrithlone (sulfarlem $^{\circledR}$ ) se discute en $3^{\text {e }}$ intension. La pilocarpine per os est prescrite en dernier recours du fait de ses effets indésirables dose dépendants, et souvent en association avec l'anétholtrithlone, de façon à en diminuer la dose.

\section{HYPERPLASIE GINGIVALE}

Certains inhibiteurs calciques comme la nifédipine peuvent provoquer l'apparition d'une hyperplasie gingivale localisée ou généralisée qui apparaît 2 à 4 mois après le début du traitement et disparaît progressivement en quelques mois à son arrêt. La gencive est molle et hémorragique. L'analyse anatomopathologique met en évidence des infiltrats inflammatoires très denses.

\section{DYSGUEUSIE}

Elle est réversible à l'arrêt du traitement et peut être provoquée par certains inhibiteurs calciques, inhibiteurs de l'enzyme de conversion. Elle se manifeste par une altération de la perception gustative au niveau des papilles linguales.

\section{RASH CUTANÉS}

Ils peuvent être observés chez des patients traités par inhibiteurs calciques, inhibiteurs de l'enzyme de conversion, alpha-bloquants.

\section{PARESTHÉSIES}

Elles peuvent apparaître au cours d'un traitement par certains diurétiques. 
- précautions dans le cadre de l'anesthésie

L'utilisation des vasoconstricteurs en chirurgie buccale est d'un apport considérable :

- ils permettent de limiter le saignement per-opératoire ;

- ils augmentent la durée d'action de l'anesthésie ;

- ils permettent l'injection de doses moindres, donc diminuent la toxicité systémique.

Ils améliorent considérablement l'efficacité de l'anesthésie.

\section{CONSÉQUENCE \\ DE L'UTILISATION \\ D'ANESTHÉSIQUES ADRÉNALINÉS}

Une fois dans la circulation, l'adrénaline agit sur certains récepteurs adrénergiques et entraîne une augmentation de la tension artérielle en stimulant la force et la vitesse des battements cardiaques.

Le risque potentiel chez le patient hypertendu est donc de déclencher une poussée hypertensive par une injection rapide et massive.

Une cartouche adrénalinée à 1/100 000 contient $17 \mathrm{mg}$ d'adrénaline ce qui correspond à la sécrétion endogène par minute du patient détendu, mais ce qui est négligeable par rapport à la sécrétion endogène du patient anxieux.

Il est donc recommandé de réaliser des injections lentes et de se limiter chez ces patients à un maximum de deux cartouches adrénalinées à 1/100 000, ou quatre cartouches adrénalinées à $1 / 200000$.

\section{INTERACTION}

\section{ADRÉNALINE / BÊTA-BLOQUANTS}

Il existe une compétition entre les deux molécules car elles agissent sur les mêmes récepteurs bêta-adrénergiques ; l'effet anti-hypertenseur du bêtabloquant peut donc être diminué.

En conséquence nous suivrons les recommandations citées plus haut.

\section{INTERACTION \\ LIDOCAIINE \\ (XYLOCAINE) / BÊTABLOQUANT}

Il a été décrit une baisse du métabolisme hépatique de la lidocaïne lors de traitements concomitants par certains bêta-bloquants (le propanolol, le métoprolol et le nadolol). Le taux plasmatique de la lidocaïne augmente donc, ce qui potentialise le risque de surdosage, avec toxicité neurologique et cardiaque. On évitera donc la xylocaïne, celle-ci étant avantageusement remplacée par d'autres molécules comme l'articaïne.

\section{INTERACTION \\ AVEC LES ANESTHÉSIQUES GÉNÉRAUX}

Les anesthésiques généraux potentialisent les antihypertenseurs et peuvent être à l'origine d'hypotensions sévères; on préfèrera donc, dans la mesure $d u$ possible, une anesthésie locale à une anesthésie générale chez ces patients. 


\section{conclusion}

L'hypertendu de plus de 65 ans est un sujet chez lequel peuvent survenir, de façon relativement prévisible, certaines complications autour d'un acte chirurgical. La bonne connaissance de notre patient et de ses traitements, ainsi que la connaissance des règles de bonne conduite nous permettront de faire les choix adéquats, en matière de plan de traitement et d'intervention.

\section{ouvrages à consulter}

Arreto CD, Gaudy JF.

Manuel d'analgésie

en odontostomatologie.

$2^{\mathrm{e}}$ éd. Paris : Masson, 2005

Arreto CD

Aspects pharmacologiques

des anesthésiques locaux utilisés

en odonto-stomatologie.

Réalités Cliniques 1991;2(1):

85-98

Dabire H, Brahimi M,

Hadj-Brahim F, Le Clesiau $\mathrm{H}$, Attali JR, Valensi P.

Rôle de la balance

vagosympathique

dans l'hypertension associée

à l'obésité.

Archives des maladies du cœur

et des vaisseaux

Tome 97. 2004;7/8:749-752.

Descroix V, Yasukawa K.

Les médicaments en odontostomatologie.

Paris : Maloine, 2005.

Ding J, Visser $M$,

Kritchevsky SB, Nevitt M,

Newman A, Sutton-Tyrrell K,

Harris TB.

The association of regional

fat depots with hypertension

in older persons of white

and African American

ethnicity.

Am J Hypertens 2004;17:10.

Franklin Stanlay S.

Systolic blood pressure:

it's time to take control.

Am J Hypertens 2004;17:12(2).
Girerd X, Digeos-Hasnier S,

Le Heuzey JY.

Guide pratique

de I'hypertension artérielle.

Collection Médiguides,

$2^{\mathrm{e}}$ éd. MMI éditions.

Paris : Masson, 2001

Girerd X.

Caractéristiques cliniques

des hypertendus

non contrôlés

suivis en médecine générale.

Rev Prat 2005;684/685(19):

305-309.

Hadida A, Slama M, Yigiroglu A

Anesthésie et pathologies

cardio-vasculaires.

Réalités Cliniques. 1991;2(1):

133-136

Hanon $\mathrm{O}$, Marquand $\mathrm{A}$,

Fauvel JP, Mounier-Vehier C,

Hottelart C, Fourcade J,

Dimitrov Y, Girerd X.

Association

entre le niveau tensionnel

en consultation et le suivi

d'un protocole d'automesure.

Archives des maladies du cœur

et des vaisseaux

Tome 97. 2004;7/8:762-766.

Laurent P.

Cardiologue, spécialiste

de I'HTA, auteur du site

www.e-cardiologie.com.

Le Charpentier Y, Auriol V.

Histopathologie

buccodentaire

et maxillofaciale.

Paris : Masson, 1997.
Neal M.

Pharmacologie médicale.

Collection «En bref».

Ed. De Boeck, 2003.

Piette, Reychler.

Traité de Pathologies

buccales et maxillofaciales.

Ed. De Boeck Université, 1991.

Philippe F, Danchin N,

Quentzel S, Cambou JP.

Utilisation des classes

thérapeutiques majeures en

prévention cardiovasculaire

chez le sujet âgé suivi en

consultation de cardiologie.

Résultats de l'enquête ELIAGE

Annales de cardiologie

et d'angéiologie

2004;53:339-346.

Postel-Vinay N.

La consultation

du sujet hypertendu.

Tome 19. Rev Prat 2005; 686/687:351-359.

Roche $Y$.

Chirurgie dentaire

et patients à risque :

évaluation et précautions

à prendre

en pratique quotidienne.

Editions Médecine-Sciences.

Paris : Flammarion, 1996.

Thaunat $\mathrm{O}$.

Athérosclérose - HTA -

Thrombose.

Modules transdisciplinaires,

Module 9.

Med-line éditions, 2003. 


\section{SUMMARY}

\section{Elderly people hypertension. Procedure to be follow during dental surgery}

H. ARNAL, C. B. WIERZBA

The prevalence of elderly people high blood pressure is important in our countries (40 to $52 \%$ ). Hypertension is an important cardio-vascular risk factor.

drugs, to decrease the level of morbidity and mortality.

The people must be treated with typotensives

The dental surgeon must know the patients and the drugs stipulates, and the side effects of the hypotensive drugs, before practicing a bloody surgery.

keywords: high blood pressure, hypertension, blood pressure, diuretics, enzymatics hypotensives drugs, calcium antagonists, beta-blockers, side effects. 